\title{
The role of reinforcement contingencies in the maintenance of vicious circle behavior
}

\author{
CYNTHIA SCHEUER \\ Florida Atlantic University, Boca Raton, Florida 33431 \\ and \\ ANTHONY P. CONSTANTINO \\ Bowling Green State University, Bowling Green, Ohio 43403
}

\begin{abstract}
Following 20 shock-escape training trials, two experimental groups of rats were reinforced either for running quickly (DRH animals could preclude shock in the center section of the runway by decreasing their starting latencies) or for running slowly (DRL animals could preclude shock in the center section of the runway by increasing their starting latencies). Results showed that the DRH animals ran consistently faster than the DRL animals, although no differences between DRH animals and traditionally punished extinction subjects were obtained. The results were discussed in terms of the existing explanations of vicious circle behavior, as well as in terms of the differential effects of manipulating the reinforcement contingencies on the maintenance of self-punitive running.
\end{abstract}

First noted by Mowrer (1947), vicious circle behavior (VCB) was manifested by having rats escape from a startbox, traverse an alley, and enter a goalbox. The subject soon learned to avoid shock by running quickly to a distinctive nonelectrified goalbox. After the escape response was well established, only a small section of the alley floor was electrified. Although the subject was then able to avoid all shock by remaining in the startbox, it continued to traverse the alley and run across the shock. In a similar study by Gwinn (1949), using an avoidance paradigm, punished extinction once again increased resistance to extinction, and the subjects were locked into VCB.

A widely accepted interpretation of $\mathrm{VCB}$, suggested by Mowrer (1947) and elaborated by Brown (1969), proposes that punished extinction subjects continue to traverse the alley and receive shock because the running response learned during training is reinforced in the goalbox by reduction of both pain and fear. Shock encountered in the alley during extinction trials results in further fear conditioning, which generalizes back to the start area. Subjects continue to leave the startbox to escape from fear and are shocked in the alley, maintaining fear, thus completing the vicious circle. Regular extinction subjects no longer encounter shock; running is no longer reinforced by pain reduction, and fear elicited by the startbox cues extinguishes.

An alternate interpretation of VCB is based on the subject's ability to discriminate between acquisition and extinction conditions (Church, 1963); it states that the

This study was a portion of a thesis submitted by the second author in partial fulfillment of the requirements for the degree of Master of Arts in psychology at Florida Atlantic University. more similar acquisition and extinction are, the less likely it is that a subject will be able to distinguish between the two conditions and, therefore, the greater will be the resistance to extinction. The relevant cue for punished extinction subjects is the presence of shock during the last training trials and the testing trials. Although both of the theories have been proposed as viable interpretations of $\mathrm{VCB}$, much of the literature fails to support the discrimination hypothesis.

At present, although the Mowrer-Brown twoprocess theory is the most popular theory for explaining the VCB phenomenon, some still feel that it is not the only viable explanation of this behavior. For example, Scheuer (Note 1) hypothesized that, during training, subjects are reinforced for running quickly by minimizing the duration of shock (i.e., the shorter the latency, the quicker the animal gets past the shock zone and, thus, the shorter the shock duration). This contingency is maintained in extinction as well. If the reinforcement for running quickly is removed by increasing shock duration independent of a subject's latency, running should extinguish quickly. To accomplish this, Scheuer increased shock duration contingent on running by confining animals in the shock segment for twice their asymptotic training latencies. The results showed that mean escape latencies across the 10 test trials were shortest for the nonconfinement group (short shock) and that the two confinement groups exposed to long shock extinguished most rapidly. Scheuer suggested that a major source of reinforcement for running was to minimize the duration of shock; however, there was some question as to the viability of a confinement procedure during extinction, as confinement per se, rather than the increase in shock duration, may have 
been acting as an additional punisher to suppress the running response. To eliminate problems associated with confinement and at the same time to evaluate the effects of reinforcement contingencies on VCB, the present study directly manipulated the reinforcing properties associated with running during extinction. The manipulations were accomplished by the use of two experimental groups. Group DRH was reinforced for running quickly; these rats could preclude shock if they increased their asymptotic starting speeds by $10 \%$. The DRL subjects were reinforced for running slowly; they could preclude shock if they reduced their starting speeds by $10 \%$. We predicted that the DRH group would run longer and faster than a standard PE group because the magnitude of reinforcement was being increased from short shock to its limiting case of no shock. The DRL group should show the least resistance to extinction compared with the other punished groups, as maximum reinforcement is contingent upon slower starting speeds.

\section{METHOD}

\section{Subjects}

The subjects were 40 male albino rats between 90 and 120 days old, purchased from the Holtzman Company. Each subject was housed individually and maintained on free food and water.

\section{Apparatus}

The apparatus consisted of a straight alley (121.9 x $11.4 \mathrm{x}$ $20.3 \mathrm{~cm})$, a goalbox (37.5 x $11.4 \times 20.3 \mathrm{~cm})$, and a twocompartment trapdoor startbox. The lower compartment of the startbox was an extension of the alley; it measured $30.5 \times 11.4 \times 20.3 \mathrm{~cm}$. The upper compartment measured $24.1 \times 11.4 \times 20.6 \mathrm{~cm}$. The trapdoor floor was hinged along the left edge $15.2 \mathrm{~cm}$ above the startbox floor. The floor of the startbox and alley were constructed of .3-cm-diam brass rods spaced $.75 \mathrm{~cm}$ apart, center to center. The walls and floor of the goalbox were constructed of wood and painted white. The walls of the startbox and runway were constructed of wood and painted flat black. All sections of the apparatus were covered with hinged Plexiglas lids.

A Grason-Stadler Model 700 shock generator was used to deliver shock. A white masking noise (approximately $78 \mathrm{~dB}$ ) was continuously present in the testing room through an 8 -ohm wallmounted speaker. Microswitches and associated equipment were used to record response measures and to program shock. Start time was defined as the time (in seconds) elapsing from the release of the trapdoor until closure of the first microswitch located $77.5 \mathrm{~cm}$ from the rear of the startbox. Total running time was defined as time elapsed from the release of the trapdoor to interruption of the second microswitch located $15 \mathrm{~cm}$ from the rear of the goalbox.

\section{Procedure}

Acquisition. All subjects received identical training consisting of 20 shockescape training trials at a rate of 10 trials/day for 2 consecutive days. The subject was placed in the upper compartment of the startbox and dropped to the grid floor, which was electrified with a $1.0-\mathrm{mA}$ shock. When the subject entered the goalbox, it remained there for an intertrial interval of $30 \mathrm{sec}$. A guillotine door prevented retracing into the alley. Subjects were given 10 trials on Day 1 of training and then were returned to the colony room until the next day. On Day 2 of training, subjects received 10 additional training trials. Subjects were tested in squads of eight per week (two per condition).
Extinction. Immediately following the last training trial, subjects were randomly assigned to one of four groups: DRL, DRH, PE, and RE. A subject assigned to the DRL group was able to preclude shock in the center $60-\mathrm{cm}$ section of the runway by reducing its starting speed (increasing its latency) $10 \%$ of the average of its last three training trials. An animal in Group DRH was able to preclude shock in the runway by increasing its starting speed (decreasing its latency) by $10 \%$. Subjects in Group PE received shock in the center section of the runway on all extinction trials. Subjects in Group RE never received shock during extinction. Any subject failing to enter the goalbox within $60 \mathrm{sec}$ on three consecutive trials was terminated from the experiment and the total number of completed runs recorded. Four subjects were replaced when they failed to leave the starting area and thus were never exposed to the avoidance contingencies.

\section{Acquisition}

\section{RESULTS}

Two one-way analyses of variance were conducted to ascertain whether terminal starting and running speeds were comparable across groups prior to the introduction of the extinction contingencies. A comparison of the mean starting latencies across groups during the final three training trials failed to achieve statistical significance $(F<1.00)$, as did a comparison of the mean running latencies across the four groups $(\mathrm{F}<1.00)$.

\section{Extinction}

Trials to criterion. Due to extensive heterogeneity of variance during the extinction trials, nonparametric tests were conducted throughout the entire analysis. A Kruskal-Wallis one-way analysis of variance by ranks was conducted across the four groups on the number of extinction trials to criterion; significant differences were found $[H(3) \geqslant 11.85, p<.02]$. As can be seen in Figure 1, Group RE had fewest median number of extinction runs (4.5), followed by Group DRL (16), DRH (33.5), and, finally, Group PE, with the greatest number of runs (38.5). Individual comparisons between groups were then performed using the Mann-Whitney U test; significant differences were found between Groups RE and DRH $(\mathrm{U}=11.00, \mathrm{p}<.001)$ and between

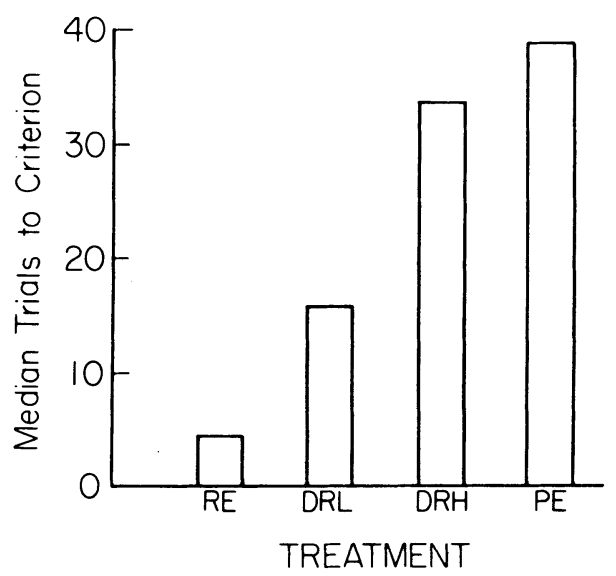

Figure 1. Median trials to criterion for each group during extinction of escape behavior. 
Groups RE and PE $(U=12.5, p<.01)$. All other comparisons were nonsignificant $(\mathrm{p}>.05)$.

Starting and running latencies. A Kruskal-Wallis one-way analysis of variance by ranks showed no significant differences between the groups' starting latencies, as can be seen in Figure 2A. When the same test was conducted on the groups' running speeds, a significant difference was found $[H(3) \geqslant 13.28, p<.01]$. As Figure 2B shows, Group DRH had the fastest median running speed, followed closely by Group PE. Group DRL ran slower than Group PE and was followed by Group RE, which had the slowest running time. When Mann-Whitney $U$ tests were performed between groups, significant differences were found between Groups RE and DRH $(U=14.00, p<.01), R E$ and PE $(\mathrm{U}=13.00, \mathrm{p}<.01), \quad \mathrm{DRL}$ and $\mathrm{DRH}(\mathrm{U}=24.00$, $\mathrm{p}<.05)$, and DRL and PE $(\mathrm{U}=19.00, \mathrm{p}<.01)$. No significant differences were found between Groups RE and DRL or DRH and PE.

Percent of avoidance and trials to criterion. A trend between the percent of avoidances and the number of trials to criterion was noticed and subsequently tested using a Spearman rank-correlation coefficient. The data for Group DRL revealed a significant negative correlation (rho $=-.62, \mathrm{p}<.05)$, and Group DRH showed a significant positive correlation $(r h o=.90, p<.01)$ between percent of avoidances and trials to criterion. These relationships revealed that, for DRL subjects, trials to criterion were inversely related to avoidance performance [i.e., the greater the percent of avoidances (reinforcement), the quicker these subjects extinguished] . In contrast, for DRH subjects, the higher the percentage of avoidances, the greater the resistance to extinction.

Starting and running latencies following avoidance trials. A Mann-Whitney U test was conducted between the percent of avoidances for Groups DRL and DRH; a significant difference was found $(U=4.5, p<.001)$. The mean number of shock avoidances for Group DRL was 68\%; the mean number of shock avoidances for Group DRH was $37 \%$. When only those subjects exposed to both of the extinction contingencies were included in

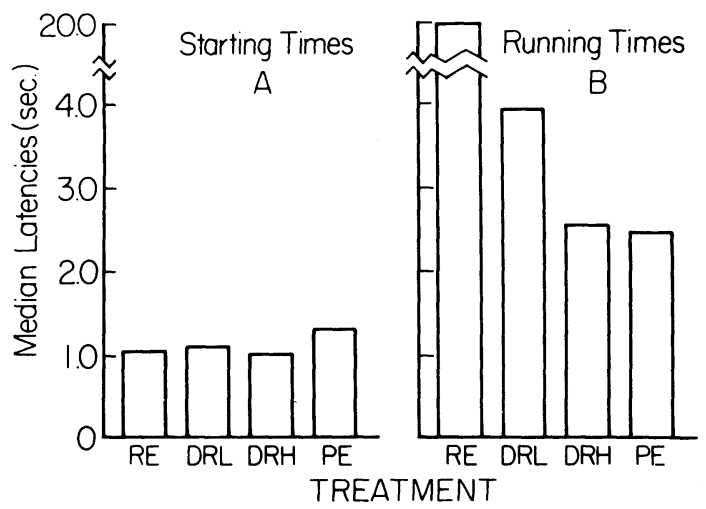

Figure 2. Median starting (A) and running (B) times for each group during extinction of escape behavior.

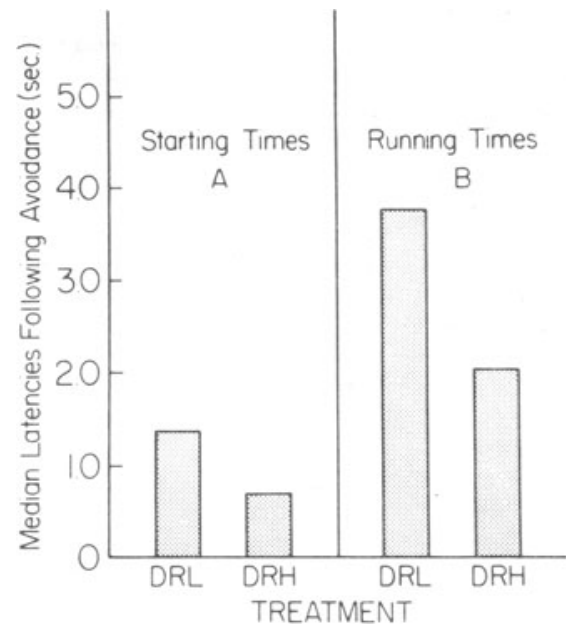

Figure 3. Median starting (A) and running (B) times for DRL and DRH subjects following successful shock-avoidance trials.

the analysis (i.e., shocked and nonshocked trials), a Mann-Whitney $U$ test again revealed a significant difference across groups $(U=4.5, p<.007)$. Both of these analyses showed that the DRL animals avoided a significantly greater number of shocks than did the DRH subjects.

An analysis was also conducted to evaluate median starting and running latencies for trials following successful avoidance trials for both DRL and DRH subjects. Again, it was meaningful to include only those subjects exposed to both extinction contingencies (shocked and nonshocked trials). Mann-Whitney $\mathrm{U}$ tests showed significant differences between starting latencies $(\mathrm{U}=5.00, \mathrm{p}<.006)$, as well as between running latencies $(\mathrm{U}=5.00, \mathrm{p}<.006)$. These differences can readily be seen in Figures $3 \mathrm{~A}$ and $3 \mathrm{~B}$. In both instances, following avoidance trials, DRH animals ran faster than the animals in the DRL group, a result consonant with their respective reinforcement contingencies.

\section{DISCUSSION}

The results of this study point out a number of interesting aspects concerning the differential effects of manipulating the reinforcement contingencies and their subsequent effects on VCB. It was expected that if reinforcement for running quickly was removed (DRL animals), running would extinguish rapidly, and this group would have the least resistance to extinction. This group, in fact, did not differ significantly from the RE group on any of the measures taken during extinction. On the other hand, DRL subjects did differ from PE subjects on each of the measures, demonstrating that once reinforcement for running quickly is removed (by punishing increases in starting speeds), subjects will, in fact, run fewer trials as well as run slower than a standard punished extinction group. Furthermore, DRL animals consistently ran slower than DRH animals, and this indicates that the avoidance contingencies differentially affected the behaviors of these two groups. DRL animals, no longer being reinforced for running quickly, began to slow down following an avoidance trial; at the same time, DRH animals ran faster following a successful avoidance trial. Furthermore, for DRL subjects, 
as exposure to the reinforcement contingencies increased (avoidance), the number of trials to extinction decreased. In other words, maximal reinforcement for decreasing running speed (i.e., zero shock duration) was gradually achieved.

We also predicted that DRH animals would show the greatest resistance to extinction, as well as the fastest starting and running latencies. DRH subjects, however, did not differ from the PE subjects on any of our measures. They did differ, however, from the RE group on each analysis. The data also revealed that, as exposure to the reinforcement contingencies increased, the number of trials to criterion also increased. This relationship was just the opposite of that found for DRL subjects; that is, maximal reinforcement for increasing running speeds for DRH subjects produces zero shock duration, thus facilitating resistance to extinction.

The highly significant correlations between percent of avoidances and trials to criterion may be related to the relative frequency of exposure to the absence of shock or to the requirements for avoidance for each of the groups. Considering the second alternative, DRL subjects may preclude shock by either slowing down or not running at all, a limiting condition of a slow rate. DRH subjects may avoid shock by either decreasing their starting latencies or, again, remaining in the start area. These two avoidance contingencies are incompatible for DRH subjects, whereas these two alternative behaviors are compatible for DRL subjects. Since extinction is considered no running for three consecutive trials, one would expect that the more frequently DRL animals are exposed to the avoidance of shock, the more quickly they will learn to remain in the start area. In contrast, the more frequently the DRH animals are exposed to avoidance of shock, the longer it will take them to avoid shock by performing a response incompatible with a high rate, that of remaining in the start area.

The present results may alternatively be viewed by assuming that resistance to extinction of VCB is simply a function of the frequency of exposure to the absence of shock. PE subjects receive shock on all test trials and are thus never exposed to a situation in which fear can be extinguished. Avoidance can be obtained only by remaining in the start area, but due to shock on the previous trial, fear is maintained and motivates running on the subsequent trial. In contrast, RE subjects can preclude shock by either running or remaining in the start area. Thus, extinction is readily achieved. DRL animals are exposed to the next highest frequency of shock nonoccurrence due to the manner in which the avoidance contingencies interact with the extinction criterion. In contrast, DRH subjects, probably because of the difficulty in increasing their starting speeds above training levels, avoid relatively few shocks but, nevertheless, do avoid some of them. The absence of shock during testing eventually permits the subjects to discriminate a change in the testing situation, and extinction takes place. Thus, the relative resistance to extinction of VCB may be directly related to the frequency of shock trials during testing. Since it is easier for DRL subjects to slow down, they eventually avoid more shocks and extinguish more rapidly than DRH animals.

Starting latencies did not differ significantly between the groups. It is possible that DRH animals may already have been at asymptote prior to extinction. Two solutions to this problem are feasible. The first would require changing the $10 \%$ requirement to any increase in speed over the mean of the last three training trials. In this way, both DRH and DRL animals would have a greater opportunity to be exposed to both contingencies. A second solution might be to initiate extinction before the subject achieves asymptote during acquisition of the escape response.

It should be pointed out that both two-process theory and the discrimination hypothesis could account for the results obtained in the present study. The two-process theory would predict relative resistance to extinction in terms of the relative shock frequency received by the four groups (i.e., the greater the shock frequency, the greater the fear and the greater the number of self-punitive runs). The discrimination hypothesis would predict relative resistance to extinction in terms of the similarity of percent of shocks occurring during acquisition and extinction (i.e., the greater the difference in percent shock, the faster the extinction and, therefore, the fewer the number of self-punitive runs).

We would like to point out, however, that it was not the contention of the present study to prove or disprove either twoprocess theory or the discrimination hypothesis as they apply to VCB. It was designed to evaluate the extent to which reinforcement contingencies contribute to the maintenance of VCB. This was accomplished by a technique that directly manipulates reinforcement for running quickly as well as for running slowly; differential behavior was found between the two experimental groups following successful avoidance trials. In conclusion, it is our contention that the avoidance contingencies for DRL subjects permitted them to be exposed to a greater number of nonshock trials than did the requirements for the DRH subjects. Thus, DRL animals had a greater opportunity to (1) extinguish fear and/or (2) discriminate that shock was not occurring in the start area.

\section{REFERENCE NOTE}

1. Scheuer, C. Precluding vicious circle behavior by removal of reinforcement for running. Paper presented at the meeting of the Midwest Psychological Association, Chicago, May 1978.

\section{REFERENCES}

Brown, J. S. Factors influencing self-punitive locomotive behavior. In B. A. Campbell \& R. M. Church (Eds.), Punishment and aversive behavior. New York: Appleton-Century-Crofts, 1969.

Church, R. M. The varied effects of punishment on behavior. Psychological Review, 1963, 70, 369-402.

Gwins, G. T. The effects of punishment on acts motivated by fear. Journal of Experimental Psychology, 1949, 39, 260-269.

Mowrer, $\mathrm{O}$. H. On the dual nature of learning-A reinterpretation of "conditioning" and "problem solving." Harvard Educational Review, 1947, 17, 102-148.

(Received for publication November 6, 1979.) 